\title{
Class of exactly solvable scattering potentials in two dimensions, entangled-state pair generation, and a grazing-angle resonance effect
}

\author{
Farhang Loran ${ }^{1, *}$ and Ali Mostafazadeh ${ }^{2, \dagger}$ \\ ${ }^{1}$ Department of Physics, Isfahan University of Technology, Isfahan 84156-83111, Iran \\ ${ }^{2}$ Departments of Mathematics and Physics, Koç University, 34450 Sarlyer, Istanbul, Turkey
}

(Received 4 October 2017; published 26 December 2017)

\begin{abstract}
We provide an exact solution of the scattering problem for the potentials of the form $v(x, y)=\chi_{a}(x)\left[v_{0}(x)+\right.$ $\left.v_{1}(x) e^{i \alpha y}\right]$, where $\chi_{a}(x):=1$ for $x \in[0, a], \chi_{a}(x):=0$ for $x \notin[0, a], v_{j}(x)$ are real or complex-valued functions, $\chi_{a}(x) v_{0}(x)$ is an exactly solvable scattering potential in one dimension, and $\alpha$ is a positive real parameter. If $\alpha$ exceeds the wave number $k$ of the incident wave, the scattered wave does not depend on the choice of $v_{1}(x)$. In particular, $v(x, y)$ is invisible if $v_{0}(x)=0$ and $k<\alpha$. For $k>\alpha$ and $v_{1}(x) \neq 0$, the scattered wave consists of a finite number of coherent plane-wave pairs $\psi_{n}^{ \pm}$with wave vector: $\mathbf{k}_{n}=\left( \pm \sqrt{k^{2}-[n \alpha]^{2}}, n \alpha\right)$, where $n=0,1,2, \ldots<k / \alpha$. This generalizes to the scattering of wave packets and suggests means for generating quantum states with a quantized component of momentum and pairs of states with an entangled momentum. We examine a realization of these potentials in terms of certain optical slabs. If $k=N \alpha$ for some positive integer $N$, $\psi_{N}^{ \pm}$coalesce and their amplitude diverge. If $k$ exceeds $N \alpha$ slightly, $\psi_{N}^{ \pm}$have a much larger amplitude than $\psi_{n}^{ \pm}$ with $n<N$. This marks a resonance effect that arises for the scattered waves whose wave vector makes a small angle with the faces of the slab.
\end{abstract}

DOI: 10.1103/PhysRevA.96.063837

\section{INTRODUCTION}

Exactly solvable models have played a central role in our understanding of the conceptual and practical aspects of quantum mechanics [1]. They are, in particular, indispensable for developing computational techniques that make the study of realistic quantum systems possible. This is why a detailed treatment of these models forms an integral part of teaching quantum mechanics. In this respect, it is quite unfortunate that the discovery of an exactly solvable model is an extremely rare event. The purpose of this article is to report such a discovery. In particular, we introduce a class of complex potentials in two dimensions whose scattering problem admits an exact and closed-form solution in terms of the elementary functions. We use this solution to offer an exact description of the scattering of polarized electromagnetic waves by certain optical slab systems. This reveals a number of intriguing properties of these systems that make them of immediate interest for engineering optical devices such as a source for coherent plane-wave pairs, quantum states with entangled momentum, states with a quantized momentum along one direction, and directional multimode lasers.

An exclusive feature of complex potentials in one dimension is their ability to display nonreciprocal reflection [2]. An extreme example is a unidirectionally invisible potential, which is reflectionless from one direction and displays perfect transmission from both directions [3-10]. The principal example is $[6,11-13]$

$$
v(x)=\mathfrak{z} e^{2 \pi i x / a} \chi_{a}(x)=\left\{\begin{array}{cc}
\mathfrak{z} e^{2 \pi i x / a} & \text { for } x \in[0, a] \\
0 & \text { otherwise }
\end{array},\right.
$$

\footnotetext{
*loran@cc.iut.ac.ir

†amostafazadeh@ku.edu.tr
}

where $\mathfrak{z}$ and $a$ are real parameters, and

$$
\chi_{a}(x):=\left\{\begin{array}{cc}
1 & \text { for } x \in[0, a] \\
0 & \text { otherwise }
\end{array}\right.
$$

To a very good approximation, this potential is invisible from the left for incident plane waves of wave number $k=\pi / a$ provided that $|\mathfrak{z}| a^{2} \ll 1$, [14].

Recently we have developed a multidimensional transfermatrix formulation of scattering theory [15] that allows for a straightforward treatment of the delta-function potentials in two and three dimensions and paves the way for a multidimensional generalization of unidirectional invisibility [16]. In this article, we use this formulation to solve the scattering problem for the potentials of the form

$$
v(x, y)=\left[v_{0}(x)+v_{1}(x) e^{i \alpha y}\right] \chi_{a}(x),
$$

where $v_{0}(x)$ and $v_{1}(x)$ are piecewise continuous real- or complex-valued functions, $v_{0}(x) \chi_{a}(x)$ is a potential whose scattering problem in one dimension is exactly solvable, and $\alpha$ and $a$ are positive real parameters. In particular, we assume that the transfer matrix $\mathbf{M}_{v_{0}}^{(1 \mathrm{~d})}$ of $v_{0}(x) \chi_{a}(x)$ in one dimension is given [17,18]. Reference [19] considers the diffraction by absorbing crystals that are modeled by a particular example of the potentials (3) with $v_{0}(x)$ and $v_{1}(x)$ being imaginary constants.

Our article is organized as follows. In Sec. II we outline the basic ingredient of transfer-matrix formulation of scattering theory in two dimensions. In Sec. III we use these to solve the scattering problem for the potentials of the form (3). In Sec. IV we discuss the physical implications, optical realizations, and potential applications of our general results. Section V includes a summary of our findings and presents our concluding remarks. 


\section{TRANSFER-MATRIX FORMULATION OF SCATTERING IN TWO DIMENSIONS}

Let $v(x, y)$ be a potential such that for $x \rightarrow \pm \infty$ the solutions of the Schrödinger equation,

$$
-\nabla^{2} \psi(x, y)+v(x, y) \psi(x, y)=k^{2} \psi(x, y),
$$

tend to plane waves:

$$
\frac{1}{2 \pi} \int_{-k}^{k} d p e^{i p y}\left[A_{ \pm}(p) e^{i \varpi(p) x}+B_{ \pm}(p) e^{-i \varpi(p) x}\right],
$$

where $k$ is a positive real wave number, $A_{ \pm}(p)$ and $B_{ \pm}(p)$ are coefficient functions vanishing for $|p|>k$, and

$$
\varpi(p):=\sqrt{k^{2}-p^{2}} .
$$

For a left-incident scattering solution of (4), $A_{-}(p)=2 \pi \delta(p)$, $B_{+}(p)=0$, and

$$
\psi(r, \theta) \rightarrow e^{i k x}+\sqrt{i / k r} e^{i k r} f(\theta) \text { as } r \rightarrow \infty,
$$

where $(r, \theta)$ are polar coordinates, and $f(\theta)$ is the scattering amplitude of $v(x, y)$. The contributions of the scattering potential to the reflected and transmitted waves are, respectively, determined by $T_{-}(p):=B_{-}(p)$ and $T_{+}(p):=A_{+}(p)-A_{-}(p)$. These by construction fulfill

$$
T_{ \pm}(p)=0 \text { for }|p|>k .
$$

We identify the transfer matrix of the potential $v(x, y)$ with the $2 \times 2$ matrix operator $\mathbf{M}(p)$ satisfying [15]

$$
\mathbf{M}(p)\left[\begin{array}{l}
A_{-}(p) \\
B_{-}(p)
\end{array}\right]=\left[\begin{array}{l}
A_{+}(p) \\
B_{+}(p)
\end{array}\right] .
$$

Its entries, $M_{i j}(p)$, are linear operators that act in the space $\mathscr{F}$ of functions $\phi(p)$ vanishing for $|p|>k . \mathbf{M}(p)$ has two basic properties: (1) it shares the composition property of its onedimensional analog [18] and (2) it has complete information about the scattering features of $v(x, y)$.

Property (1) follows from the fact that

$$
\mathbf{M}(p)=\mathscr{T} \exp \int_{-\infty}^{\infty} d x[-i \mathbf{H}(x, p)],
$$

where $\mathscr{T}$ stands for the time-ordering operation with $x$ playing the role of time, $\mathbf{H}(x, p)$ is an effective non-Hermitian operator given by

$$
\mathbf{H}(x, p):=\frac{1}{2 \varpi(p)} e^{-i \varpi(p) x \sigma_{3}} v\left(x, i \partial_{p}\right) \mathcal{K} e^{i \varpi(p) x \sigma_{3}},
$$

$\sigma_{i}$ are the Pauli matrices, $\mathcal{K}:=\sigma_{3}+i \sigma_{2}, v\left(x, i \partial_{p}\right)$ is the linear operator acting in $\mathscr{F}$ according to

$$
v\left(x, i \partial_{p}\right) \phi(p):=\frac{1}{2 \pi} \int_{-k}^{k} d q \tilde{v}(x, p-q) \phi(q),
$$

and $\tilde{v}\left(x, \mathfrak{K}_{y}\right)$ denotes the Fourier transform of $v(x, y)$ with respect to $y$, i.e., $\tilde{v}\left(x, \mathfrak{K}_{y}\right):=\int_{-\infty}^{\infty} d y e^{-i \mathfrak{K}_{y} y} v(x, y)$.
Property (2) is a result of the following relations [15]:

$$
\begin{gathered}
T_{-}(p)=-2 \pi M_{22}(p)^{-1} M_{21}(p) \delta(p), \\
T_{+}(p)=M_{12}(p) T_{-}(p)+2 \pi\left[M_{11}(p)-1\right] \delta(p), \\
f(\theta)=-\frac{i k|\cos \theta|}{\sqrt{2 \pi}} \times \begin{cases}T_{-}(k \sin \theta) & \text { for } \cos \theta<0 \\
T_{+}(k \sin \theta) & \text { for } \cos \theta \geqslant 0\end{cases}
\end{gathered}
$$

\section{SOLUTION OF THE SCATTERING PROBLEM}

The calculation of the effective Hamiltonian (9) for the potentials of the form (3) is straightforward. First, we substitute (3) in (10) to show that

$$
v\left(x, i \partial_{p}\right)=\chi_{a}(x)\left[v_{0}(x)+v_{1}(x) S_{\alpha}\right],
$$

where $S_{\alpha}$ is the shift operator defined by

$$
S_{\alpha} \phi(p):=\phi(p-\alpha) .
$$

In view of (9) and (56),

$$
\begin{gathered}
\mathbf{H}(x, p)=\mathbf{H}_{0}(x, p)+\mathbf{H}_{1}(x, p) S_{\alpha}, \\
\mathbf{H}_{j}(x, p):=\frac{v_{j}(x) \chi_{a}(x)}{2 \varpi(p)} e^{-i \varpi(p) x \sigma_{3}} \mathcal{K} e^{i \varpi(p-j \alpha) x \sigma_{3}},
\end{gathered}
$$

where $j=0,1$. Because $\mathbf{H}(x, p)$ vanishes for $x \notin[0, a]$, we can replace $-\infty$ and $\infty$ in (8) by 0 and $a$, respectively.

Next, we separate the contribution of $v_{0}(x)$ to the transfer matrix $\mathbf{M}(p)$ of $v(x)$. To this end we introduce

$$
\begin{array}{r}
\mathbf{M}_{0}(x, p):=\mathscr{T} \exp \int_{0}^{x} d x^{\prime}\left[-i \mathbf{H}_{0}\left(x^{\prime}, p\right)\right], \\
\widehat{\mathbf{H}}_{1}(x, p):=\mathbf{M}_{0}(x, p)^{-1} \mathbf{H}_{1}(x, p) \mathbf{M}_{0}(x, p-\alpha), \\
\widehat{\mathbf{M}}(p):=\mathscr{T} \exp \int_{0}^{a} d x\left[-i \widehat{\mathbf{H}}_{1}(x, p) S_{\alpha}\right],
\end{array}
$$

so that $\mathbf{M}_{0}(p):=\mathbf{M}_{0}(\infty, p)=\mathbf{M}_{0}(a, p)$ is the transfer matrix of the potential $v_{0}(x) \chi_{a}(x)$ in two dimensions, and

$$
\mathbf{M}(p)=\mathbf{M}_{0}(p) \widehat{\mathbf{M}}(p) .
$$

This reduces the calculation of $\mathbf{M}(p)$ to that of $\mathbf{M}_{0}(p)$ and $\widehat{\mathbf{M}}(p)$.

The determination of $\mathbf{M}_{0}(p)$ is equivalent to finding the transfer matrix of $v_{0}(x) \chi_{a}(x)$ in one dimension [14] and substituting $\varpi(p)$ for the wave number $k$ in the result:

$$
\mathbf{M}_{0}(p)=\left.\mathbf{M}_{v_{0}}^{(1 \mathrm{~d})}\right|_{k \rightarrow \varpi(p)} .
$$

In particular $\mathbf{M}_{0}(p)$ is a known $2 \times 2$ matrix with unit determinant. Notice also that

$$
\mathbf{M}_{0}(x, p)=\left.\mathbf{M}_{0}(p)\right|_{a \rightarrow x}=\left.\mathbf{M}_{v_{0}}^{(1 \mathrm{~d})}\right|_{k \rightarrow \varpi(p), a \rightarrow x} .
$$

To calculate $\widehat{\mathbf{M}}(p)$ we use (15) and the definition of the timeordered exponential to write (20) in the form

$$
\widehat{\mathbf{M}}(p)=\mathbf{I}+\sum_{n=1}^{\infty} \widehat{\mathbf{M}}_{n}(p) S_{n \alpha},
$$


Here $\mathbf{I}$ is the $2 \times 2$ identity matrix, and

$$
\begin{aligned}
& \widehat{\mathbf{M}}_{n}(p):=(-i)^{n} \int_{0}^{a} d x_{n} \int_{0}^{x_{n}} d x_{n-1} \cdots \int_{0}^{x_{2}} d x_{1} \mathcal{H}_{n}, \\
\mathcal{H}_{n}:= & \widehat{\mathbf{H}}_{1}\left(x_{n}, p\right) \widehat{\mathbf{H}}_{1}\left(x_{n-1}, p-\alpha\right) \\
& \times \widehat{\mathbf{H}}_{1}\left(x_{n-2}, p-2 \alpha\right) \cdots \widehat{\mathbf{H}}_{1}\left(x_{1}, p-[n-1] \alpha\right) .
\end{aligned}
$$

Now consider an incident wave with wave number $k$ and denote the integer part of $k / \alpha$ by $N(k)$. Then, according to (15), for any test function $\phi(p)$ that vanishes for $p<-k$, $S_{[N(2 k)+1] \alpha} \phi(p)=0$ for all $p$. Combining this relation with the fact that $A_{-}(p)=B_{-}(p)=0$ for $p<-k$ and using (7) and (21), we see that the terms on the right-hand side of (24) vanish for $n>N(2 k)$, i.e., $\widehat{\mathbf{M}}(p)=\mathbf{I}+\sum_{n=1}^{N(2 k)} \widehat{\mathbf{M}}_{n}(p) S_{n \alpha}$. As a result, (21) gives

$$
\mathbf{M}(p)=\sum_{n=0}^{N(2 k)} \mathbf{M}_{n}(p) S_{n \alpha}
$$

where

$$
\mathbf{M}_{n}(p):=\mathbf{M}_{0}(p) \widehat{\mathbf{M}}_{n}(p) \text { for } n \geqslant 1
$$

According to (27) the entries of $\mathbf{M}(p)$ have the form

$$
M_{i j}(p)=M_{i j}^{(0)}(p)+\sum_{n=1}^{N(2 k)} M_{i j}^{(n)}(p) S_{n \alpha},
$$

where $M_{i j}^{(n)}(p)$ are the entries of $\mathbf{M}_{n}(p)$. It is important to note that these are functions of $p$ that act on other functions of $p$ by multiplication.

In order to determine the scattering amplitude $f(\theta)$ for the potentials (3) we compute the reflection and transmission functions $T_{ \pm}(p)$. To do this we first write (11) in the form

$$
M_{22}(p) T_{-}(p)=-2 \pi M_{21}(p) \delta(p)
$$

and propose the following ansatz for its solution:

$$
T_{-}(p)=2 \pi \sum_{n=0}^{N(k)} t_{n}^{-} \delta(p-n \alpha)
$$

where $t_{n}^{-}$are coefficients that we determine by inserting (31) in (30) and making use of (29) and (6). The result is the following iterative construction of $t_{n}^{-}$:

$$
\begin{gathered}
t_{0}^{-}=-\frac{M_{21}^{(0)}(0)}{M_{22}^{(0)}(0)}, \\
t_{\ell}^{-}=-\frac{M_{21}^{(\ell)}(\ell \alpha)+\sum_{m=1}^{\ell} M_{22}^{(m)}(\ell \alpha) t_{\ell-m}^{-}}{M_{22}^{(0)}(\ell \alpha)}
\end{gathered}
$$

where $\ell=1,2, \ldots, N(k)$. Remarkably, $t_{0}^{-}$coincides with the left reflection amplitude $R^{1}$ for the potential $v_{0}(x) \chi_{a}(x)$ in one dimension [17].

We can view (32) and (33) as a linear system of $N^{\prime}:=$ $N(k)+1$ equations for $t_{n}^{-}$. Identifying the latter with the entries of a column vector $\mathbf{t}^{-}$, we can write the solution of these equations as $\mathbf{t}^{-}=\sum_{n=0}^{N(k)} \mathbf{A}^{n} \mathbf{b}$, where $\mathbf{A}$ and $\mathbf{b}$ are, respectively, the $N^{\prime} \times N^{\prime}$ and $N^{\prime} \times 1$ matrices with entries:

$$
\begin{aligned}
& A_{n n^{\prime}}:=\left\{\begin{array}{cc}
-\frac{M_{22}^{\left(n-n^{\prime}\right)}(n \alpha)}{M_{22}^{(0)}(n \alpha)} & \text { for } 0 \leqslant n^{\prime} \leqslant n-1 \\
0 & \text { otherwise }
\end{array},\right. \\
& b_{n}:=-\frac{M_{21}^{(n)}(n \alpha)}{M_{22}^{(0)}(n \alpha)}, \quad n, n^{\prime}=0,1,2, \ldots, N(k) .
\end{aligned}
$$

Next, we use (31) to write (12) in the form

$$
T_{+}(p)=2 \pi \sum_{n=0}^{N(k)} t_{n}^{+} \delta(p-n \alpha),
$$

where

$$
\begin{gathered}
t_{0}^{+}:=M_{12}^{(0)}(0) t_{0}^{-}+M_{11}^{(0)}(0)-1=\frac{1}{M_{22}^{(0)}(0)}-1, \\
t_{\ell}^{+}:=M_{11}^{(\ell)}(\ell \alpha)+\sum_{m=0}^{\ell} M_{12}^{(\ell-m)}(\ell \alpha) t_{m}^{-} .
\end{gathered}
$$

The second equality in (35) follows from the fact that $\operatorname{det} \mathbf{M}_{0}(0)=1$. Note also that $t_{0}^{+}$coincides with $T-1$ where $T$ is the transmission amplitude for the potential $v_{0}(x) \chi_{a}(x)$ in one dimension [17].

Having computed $T_{ \pm}(p)$, we use (13) to obtain the following expression for the scattering amplitude:

$$
f(\theta)=-i \sqrt{2 \pi} \sum_{n=0}^{N(k)}\left[t_{n}^{+} \delta\left(\theta-\theta_{n}^{+}\right)+t_{n}^{-} \delta\left(\theta-\theta_{n}^{-}\right)\right],
$$

where $\theta \in[-\pi / 2,3 \pi / 2)$ and

$$
\theta_{n}^{+}:=\arcsin \left(\frac{n \alpha}{k}\right) \in\left[0, \frac{\pi}{2}\right], \theta_{n}^{-}:=\pi-\theta_{n}^{+} \in\left[\frac{\pi}{2}, \pi\right] \text {. }
$$

This completes the solution of the scattering problem for the potentials (3). To summarize, we list its basic steps:

(1) For a given $k$, determine $N(k)$, which is the integer part of $k / \alpha$, and do the following for all $n=0,1,2, \ldots, N(k)$

(2) Calculate $\mathbf{H}_{1}, \widehat{\mathbf{H}}_{1}, \mathcal{H}_{n}$, and $\widehat{\mathbf{M}}_{n}(p)$ using (17), (19), (26), and (25)

(3) Find $\mathbf{M}_{n}(p)$ and its entries $M_{i j}^{(n)}(p)$ using (28)

(4) Determine $t_{n}^{ \pm}$and $f(\theta)$ using (32)-(37).

\section{PHYSICAL IMPLICATIONS AND POTENTIAL APPLICATIONS}

The $n=0$ term on the right-hand side of (37) gives the contribution of $v_{0}(x)$ to the scattering amplitude. For $k<\alpha$, this is the only term contributing to $f(\theta)$. Therefore, the presence of $v_{1}(x)$ does not affect the scattering process. In particular, a potential of the form $v_{1}(x) e^{i \alpha y} \chi_{a}(x)$ does not scatter any incident plane wave with wave vector pointing along positive $x$ axis and $k<\alpha$, i.e., it is invisible for these waves [20].

For $k \geqslant \alpha$, the scattered wave consists of $N(k)$ pairs of plane waves $\psi_{n}^{ \pm}$propagating along the direction given by $\theta_{n}^{ \pm}$; their wave vector has the form $\left( \pm \sqrt{k^{2}-[n \alpha]^{2}}, n \alpha\right),[21]$. In particular, they have the same $y$ component, which is 
quantized in units of $\alpha$, while their $x$ component are equal in magnitude and opposite in sign. This observation, which might find applications in quantum metrology, also applies to wave packet scattering.

Consider an incident wave packet whose momentum is directed along the $x$ axis and has a spread about its average value that is much smaller than $\alpha$. The scattering of such a wave packet by a potential of the form (3) leads to scattered wave-packet pairs that are sharply peaked in the momentum space. The $y$ component of the momentum of the members of each pair are identical and quantized in units of $\alpha$, while their $x$ component adds up to zero. These correspond to a quantum state with entangled momentum. Potentials (3) may, therefore, serve as sources for entangled states.

The scattering features of the potentials (3) is reminiscent of the photoelectric effect. The incident wave and the resulting pairs of scattered waves, respectively, play the role of an incident photon on a metallic plate and the emitted electrons by the plate. Similarly to the photoelectric effect, a scattered wave appears only if the energy of the incident wave exceeds a critical value.

Next, we examine a simple optical realization of the potentials of the form (3). Consider an infinite optical slab placed between the planes $x=0$ and $x=a$ in three dimensions, and suppose that its relative permittivity $\hat{\varepsilon}$ does not depend on $z$. Let $\mathbf{e}_{j}$ be the unit vector pointing along the $j$ axis for $j=x, y, z$. The scattering of a normally incident $z$-polarized TE wave, $E_{0} e^{i k(x-c t)} \mathbf{e}_{z}$, by this slab is described by the Schrödingier equation (4) for the optical potential: $v(x, y)=k^{2}[1-\hat{\varepsilon}(x, y)]$ [16]. If this potential has the form (3), the scattering problem for these TE waves is equivalent to the one we have treated above. In particular, we can use (5) and (37) to derive the following asymptotic expression for the time-averaged Poynting vector for the scattered TE waves [16]:

$$
\langle\mathbf{S}\rangle=\frac{\left|E_{0}\right|^{2}}{2 \mu_{0} \mathrm{c}}\left\{\begin{array}{l}
\left(1+\left|t_{0}^{-}\right|^{2}\right) \mathbf{e}_{0}^{-}+\sum_{n=1}^{N}\left|t_{n}^{-}\right|^{2} \mathbf{e}_{n}^{-} \text {for } x \rightarrow-\infty \\
\left|1+t_{0}^{+}\right|^{2} \mathbf{e}_{0}^{+}+\sum_{n=1}^{N}\left|t_{n}^{+}\right|^{2} \mathbf{e}_{n}^{+} \text {for } x \rightarrow \infty
\end{array}\right.
$$

where $\mu_{0}$ and $\mathrm{c}$ are, respectively, the permeability and speed of light in vacuum, and $\mathbf{e}_{n}^{ \pm}:=\cos \theta_{n}^{ \pm} \mathbf{e}_{x}+\sin \theta_{n}^{ \pm} \mathbf{e}_{y}$.

The slab we have introduced involves a particular periodic modulation along the $y$ direction, but there is no restriction on the choice of $v_{0}(x)$ and $v_{1}(x)$, except that $v_{0}(x) \chi_{a}(x)$ must be exactly solvable in one dimension. This is a great advantage. In contrast to the unidirectionally invisible systems [6,7] modeled by the potentials of the form (1), which require balanced regions of gain and loss, we can consider a lossy slab by taking $v_{0}(x)$ to have a positive imaginary part larger than $\left|v_{1}(x)\right|$. For example, let $v_{j}(x)=-k^{2} \mathfrak{z}_{j}$, where $\mathfrak{z}_{j}$ are complex numbers such that $0<\left|\mathfrak{z}_{1}\right|<\operatorname{Im}\left(\mathfrak{z}_{0}\right)$. Then

$$
\hat{\varepsilon}(x, y)=1+\left(\mathfrak{z}_{0}+\mathfrak{z}_{1} e^{i \alpha y}\right) \chi_{a}(x)
$$

and the slab does not involve any gain regions. This is reminiscent of the concept of passive $\mathcal{P} \mathcal{T}$ symmetry [22].

For an incident wave with $k \in(\alpha, 2 \alpha), N=1$ and the scattered wave is determined in terms of $t_{0}^{ \pm}$and $t_{1}^{ \pm}$. As we show in the Appendix, for a slab described by the permittivity profile (39) we can compute these in closed form. In particular,
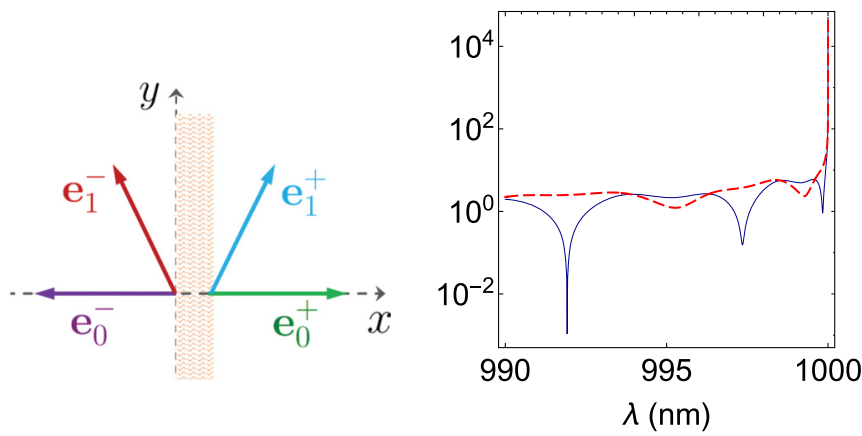

FIG. 1. Left-hand panel shows a schematic view of the directions $\mathbf{e}_{n}^{ \pm}$along which a left-incident wave with $k \in(\alpha, 2 \alpha)$ scatters by a slab described by the permittivity profile (39). Right-hand panel shows the graphs of $t_{1}^{-} / \mathfrak{z}_{1}$ (solid navy curve) and $t_{1}^{+} / \mathfrak{z}_{1}$ (red dashed curve) as functions of the wavelength $\lambda$ for a lossy titanium-sapphire slab with $a=10 \mu \mathrm{m}, \mathfrak{n}=2.892+i\left(4.5 \times 10^{-5}\right)$, and $\lambda_{\alpha}=2 \pi / \alpha=$ $1000 \mathrm{~nm}$. For the selected range of values of $\lambda, \theta_{1}^{+} \in\left[82^{\circ}, 90^{\circ}\right]$.

we have

$$
t_{1}^{ \pm}=\frac{\mathfrak{z}_{1} k^{3} A^{ \pm}}{\mathfrak{n}^{2} \alpha^{2} \varpi_{1} B},
$$

where $\mathfrak{n}:=\sqrt{1+\mathfrak{z}_{0}}$ is the refractive index of the unmodulated slab, $\varpi_{n}:=\sqrt{k^{2}-(n \alpha)^{2}}=k \cos \theta_{n}^{+}$:

$$
\begin{aligned}
A^{-}:= & -k^{-1}\left\{k_{-}\left[1-\cos \left(a k_{+} \mathfrak{n}\right)\right]\right. \\
& \left.+i\left[\mathfrak{n} \_k_{+} \sin \left(a k_{-} \mathfrak{n}\right)+\mathfrak{n}_{+} k_{-} \sin \left(a k_{+} \mathfrak{n}\right)\right]\right\}, \\
A^{+}:= & k^{-1} e^{-i a \varpi_{1}}\left\{k_{+}\left[\cos (a k \mathfrak{n})-\cos \left(a \varpi_{1} \mathfrak{n}\right)\right]\right. \\
& +i\left[\left(\mathfrak{n}^{-1} k+\mathfrak{n} \varpi_{1}\right) \sin \left(a \varpi_{1} \mathfrak{n}\right)\right. \\
& \left.\left.\quad-\left(\mathfrak{n} k+\mathfrak{n}^{-1} \varpi_{1}\right) \sin (a k \mathfrak{n})\right]\right\}, \\
B:= & \mathfrak{n}_{-}^{2} \cos \left(a k_{-} \mathfrak{n}\right)-\left(\mathfrak{n}_{+}^{2}+1\right) \cos \left(a k_{+} \mathfrak{n}\right)+2 i \mathfrak{n}_{+} \sin \left(a k_{+} \mathfrak{n}\right), \\
k_{ \pm}:= & k \pm \varpi_{1}, \text { and } \mathfrak{n}_{ \pm}=\left(\mathfrak{n} \pm \mathfrak{n}^{-1}\right) / 2 .
\end{aligned}
$$

Figure 1 shows the plots of $\mathfrak{z}_{1}^{-1}\left|t_{1}^{ \pm}\right|$as functions of the wavelength $\lambda:=2 \pi / k$ for a titanium-sapphire slab with thickness $a=10 \mu \mathrm{m}$ and modulation period $\lambda_{\alpha}:=2 \pi / \alpha=1 \mu \mathrm{m}$. As seen from this figure, the critical wavelength $\lambda=\lambda_{\alpha}$ is a singularity of $\left|t_{1}^{ \pm}\right|$. It is indeed easy to show that for $k=\alpha+\delta k$ with $0<\delta k \ll \alpha, \theta_{1}^{ \pm} \approx 90^{\circ} \mp \delta \theta$ where $\delta \theta:=\sqrt{2 \delta k / \alpha} \ll 1$, and $t_{1}^{ \pm} \approx-\mathfrak{z}_{1}\{\mathfrak{n}[\mathfrak{n}+i \cot (a \alpha \mathfrak{n} / 2)] \delta \theta\}^{-1}$. This shows that the oblique scattered waves tend to have a much larger amplitude when the wavelength of the incident wave is close to the period of the modulation $\lambda_{\alpha}$. The same holds for the scattered waves $\psi_{N}^{ \pm}$when $\lambda$ approaches $\lambda_{\alpha} / N$ for some positive integer $N$. This is a resonance effect that should be experimentally observable. Remarkably it is present also for a purely lossy slab. It is easy to show that this effect has its root in the fact that in general $t_{n}^{ \pm}=\mathfrak{z}_{1}^{n} \alpha \hat{t}_{n}^{ \pm} / \varpi_{n}$ where $\hat{t}_{n}^{ \pm}$take a nonzero value for $k \approx n \alpha$. For values of $k$ close to $N \alpha, \varpi_{N} \ll \alpha$ which implies that $\left|t_{N}^{ \pm}\right| \gg\left|\mathfrak{z}_{1}\right|^{n-N}\left|t_{n}^{ \pm}\right|$for $n<N$

Next, suppose that the slab contains gain regions. We can arrange its parameters so that for some $k>n \alpha$ and $n \geqslant 1$, $M_{22}^{(0)}(n \alpha)$ vanishes and $\left|t_{n}^{ \pm}\right|$blow up. This corresponds to the emergence of a spectral singularity [17]. It signals the onset of lasing in the TE mode of the slab with incidence (emission) angle $\theta_{n}^{ \pm}$. For the permittivity profile (39), we can easily compute $M_{22}^{(0)}(n \alpha)$ and impose $M_{22}^{(0)}(n \alpha)=0$ to obtain the threshold 
gain $g_{\star}$ and the lasing modes $k_{\star}$, [23]. For typical high-gain material, where $|\operatorname{Im}(\mathfrak{n})| \ll \operatorname{Re}(\mathfrak{n})-1 \ll a k_{\star}$, this gives

$$
\begin{gathered}
g_{\star}=\frac{4 \sqrt{\eta^{2}-\sin ^{2} \theta_{n}^{+}}}{\eta a} \ln \left[\frac{\sqrt{\eta^{2}-\sin ^{2} \theta_{n}^{+}}+\left|\cos \theta_{n}^{+}\right|}{\sqrt{\eta^{2}-1}}\right], \\
k_{\star}=\sqrt{(\pi m / \eta a)^{2}+(\alpha n)^{2}}=n \alpha / \sin \theta_{n}^{+}, \\
\theta_{n}^{+}=\arcsin \left[1 / \sqrt{1+(\pi q / \eta \alpha a)^{2}}\right],
\end{gathered}
$$

where $\eta:=\operatorname{Re}(\mathfrak{n}), \quad m=1,2,3, \ldots$ is a mode number, $q:=m / n$, and we have used (38).

According to (38)-(43), $\theta_{n}^{ \pm}$and consequently $g_{\star}$ depend on $q$, while $k_{\star}$ is a function of both $n$ and $q$. To make this more transparent we use $\theta^{ \pm}(q), g_{\star}(q)$, and $k_{n}(q)$ for $\theta_{n}^{ \pm}, g_{\star}$, and $k_{\star}$, respectively. If we attain the threshold gain $g_{\star}(q)$ for a particular value of $q$, the slab will begin emitting laser light in the directions given by $\theta^{ \pm}(q)$ and wave numbers $k_{n}(q)=n k_{1}(q)$ for different values of $n$, i.e., it functions as a directional laser with a degenerate set of lasing modes. Clearly this argument ignores the effects of dispersion and does not ensure the presence of the nonlinear effects that are necessary for having stable laser oscillators. Yet it reveals the practical importance of the longitudinal mode number $n$ that stems from the periodic modulation along the $y$ direction. This is an active analog of the grating component of the distributed feedback lasers. But unlike the latter its role is to produce an additional mode number that might find interesting applications in designing realistic multimode lasers [24].

\section{CONCLUDING REMARKS}

The discovery of an exactly solvable class of potentials is a very rare event in the history of quantum physics. Here we report such a discovery and discuss some of its potential applications. In particular, we draw attention to the relevance of our findings for the production of entangled states with a quantized $y$ component of momentum. We also outline a simple optical realization of our potentials and elaborate on their potential application in generating degenerate lasing modes. We also reveal a resonance effect arising for wave numbers that are integer multiples of $\alpha$. This leads to scattering waves of large amplitude traveling at grazing angle. It turns out that the singular behavior of these waves is regularized when one considers a slab with a finite extension along the $y$ axis. A detailed study of this effect is a subject of future investigation.

Our results can be easily generalized to the treatment of scattering of oblique incident waves. As explained in Ref. [25], this amounts to replacing $\delta(p)$ in (11) and (12) by $\delta(p-$ $\left.p_{0}\right)$, where $p_{0}$ is the $y$ component of the wave vector for the incidence wave. Another possible extension is the solution of the scattering problem for the potentials of the form $v(x)=$ $\left[v_{0}(x)+\sum_{j} v_{j}(x) e^{i \alpha_{j} y}\right] \chi_{a}(x)$ with $\alpha_{j}>0$.

\section{ACKNOWLEDGMENTS}

We are indebted to L. Ge, V. Karimipour, H.-R. Ramazani, A. Sennaroğlu, and A. Serpengüzel for fruitful discussions. This project was supported by the Turkish Academy of Sciences (TÜBA).

\section{APPENDIX}

In the following we give the details of the calculation of $t_{0}^{ \pm}$ and $t_{1}^{ \pm}$for the permittivity profile (39).

According to Eqs. (32), (33), (35), and (36),

$$
\begin{aligned}
t_{0}^{-} & =-\frac{M_{21}^{(0)}(0)}{M_{22}^{(0)}(0)}, \quad t_{0}^{+}=\frac{1}{M_{22}^{(0)}(0)}-1, \\
t_{1}^{-} & =-\frac{M_{21}^{(1)}(\alpha)+M_{22}^{(1)}(\alpha) t_{0}^{-}}{M_{22}^{(0)}(\alpha)}, \\
t_{1}^{+} & =M_{11}^{(1)}(\alpha)+M_{12}^{(1)}(\alpha) t_{0}^{-}+M_{12}^{(0)}(\alpha) t_{1}^{-},
\end{aligned}
$$

where $M_{i j}^{(n)}(p)$ are the entries of $\mathbf{M}_{n}(p)$.

For the permittivity profile (38),

$$
v_{j}(x)=-k^{2} \mathfrak{z}_{j} \chi_{a}(x), \quad j=0,1 .
$$

In particular the expression for $\mathbf{M}_{0}(p)$ follows from the formula for the transfer matrix of a homogenous slab. It has the form [15]

$$
\mathbf{M}_{0}(p)=\left[\begin{array}{cc}
F_{0}(a, \varpi) & G_{0}(a, \varpi) \\
G_{0}(a,-\varpi) & F_{0}(a,-\varpi)
\end{array}\right],
$$

where

$$
\begin{aligned}
& F_{0}(a, \varpi):=\left[\cos (a \varpi \mathfrak{n})+i \mathfrak{n}_{+} \sin (a \varpi \mathfrak{n})\right] e^{-i a \varpi}, \\
& G_{0}(a, \varpi):=i \mathfrak{n}_{-} \sin (a \varpi \mathfrak{n}) e^{-i a \varpi}, \\
& \varpi:=\varpi(p):=\sqrt{k^{2}-p^{2}} \\
& \mathfrak{n}:=\sqrt{1+\mathfrak{z}_{0}}, \quad \mathfrak{n}_{ \pm}:=\frac{\mathfrak{n}^{2} \pm 1}{2 \mathfrak{n}} .
\end{aligned}
$$

In view of (A4),

$$
\begin{array}{ll}
M_{11}^{(0)}(\alpha)=F_{0}\left(a, \varpi_{1}\right), & M_{12}^{(0)}(\alpha)=G_{0}\left(a, \varpi_{1}\right), \\
M_{21}^{(0)}(\alpha)=G_{0}\left(a,-\varpi_{1}\right), & M_{22}^{(0)}(\alpha)=F_{0}\left(a,-\varpi_{1}\right),
\end{array}
$$

where $\varpi_{1}:=\varpi(\alpha)=\sqrt{k^{2}-\alpha^{2}}=k \cos \theta_{1}^{+}$. Equations (A5) together with (A1) imply

$$
t_{0}^{-}=-\frac{G_{0}(a,-k)}{F_{0}(a,-k)}, \quad t_{0}^{+}=\frac{1}{F_{0}(a,-k)}-1 .
$$

In order to compute $t_{1}^{ \pm}$we need to determine $\mathbf{M}_{1}(\alpha)$. First, we recall that according to Eqs. (28), (25), (26), (19), (23), (A4), and (17),

$$
\begin{aligned}
\mathbf{M}_{1}(\alpha) & =\mathbf{M}_{0}(\alpha) \widehat{\mathbf{M}}_{1}(\alpha), \\
\widehat{\mathbf{M}}_{1}(\alpha) & =-i \int_{0}^{a} d x \widehat{\mathbf{H}}_{1}(x, \alpha), \\
\widehat{\mathbf{H}}_{1}(x, \alpha) & =\mathbf{M}_{0}(x, \alpha)^{-1} \mathbf{H}_{1}(x, \alpha) \mathbf{M}_{0}(x, 0), \\
\mathbf{M}_{0}(x, \alpha) & =\left[\begin{array}{cc}
F_{0}\left(x, \varpi_{1}\right) & G_{0}\left(x, \varpi_{1}\right) \\
G_{0}\left(x,-\varpi_{1}\right) & F_{0}\left(x,-\varpi_{1}\right)
\end{array}\right], \\
\mathbf{H}_{1}(x, \alpha) & =\frac{v_{1}(x) \chi_{a}(x)}{2 \varpi_{1}} e^{-i \varpi_{1} x \sigma_{3}} \mathcal{K} e^{i k x \sigma_{3}} \\
& =\frac{v_{1}(x) \chi_{a}(x)}{2 \varpi_{1}}\left[\begin{array}{cc}
e^{i k_{-} x} & e^{-i k_{+} x} \\
-e^{i k_{+} x} & -e^{-i k_{-} x}
\end{array}\right],
\end{aligned}
$$


where $k_{ \pm}:=k \pm \varpi_{1}$. Next, we substitute (A10) and (A11) in (A9) and simplify the resulting expression to obtain

$$
\widehat{\mathbf{H}}_{1}(x, \alpha)=\frac{v_{1}(x) \chi_{a}(x)}{2 \varpi_{1} \mathfrak{n}}\left[\begin{array}{cc}
h\left(k_{+}, k_{-}\right) & h\left(-k_{-},-k_{+}\right) \\
-h\left(k_{-}, k_{+}\right) & -h\left(-k_{+},-k_{-}\right)
\end{array}\right],
$$

where

$$
h(\mu, \nu):=\mathfrak{n}_{+} \cos (\nu \mathfrak{n} x)+\mathfrak{n}_{-} \cos (\mu \mathfrak{n} x)+i \sin (\nu \mathfrak{n} x) .
$$

In view of (A3) and (A12) we can evaluate the integral in (A8). Substituting the result in (A7) and using (A4), we obtain

$$
\mathbf{M}_{1}(\alpha)=\left[\begin{array}{cc}
F_{1}\left(k, \varpi_{1}\right) & F_{1}\left(-k, \varpi_{1}\right) \\
F_{1}\left(k,-\varpi_{1}\right) & F_{1}\left(-k,-\varpi_{1}\right)
\end{array}\right],
$$

where

$$
\begin{aligned}
F_{1}(\mu, \nu):= & \frac{\mathfrak{z}_{1} \mu^{2} e^{-i a v}}{2 \mathfrak{n}^{3}\left(\mu^{2}-v^{2}\right) v}\{\mathfrak{n}(\mu+v)[\cos (a \mu \mathfrak{n})-\cos (a \nu \mathfrak{n})] \\
& \left.+i\left[\left(\mu \mathfrak{n}^{2}+v\right) \sin (a \mu \mathfrak{n})-\left(\mu+\nu \mathfrak{n}^{2}\right) \sin (a \nu \mathfrak{n})\right]\right\}
\end{aligned}
$$

According to (A13),

$$
\begin{aligned}
& M_{11}^{(1)}(\alpha)=F_{1}\left(k, \varpi_{1}\right), \quad M_{12}^{(1)}(\alpha)=F_{1}\left(-k, \varpi_{1}\right), \\
& M_{21}^{(1)}(\alpha)=F_{1}\left(k,-\varpi_{1}\right), \quad M_{22}^{(1)}(\alpha)=F_{1}\left(-k,-\varpi_{1}\right) .
\end{aligned}
$$

If we insert (A6) and (A14) in (A2), we find that $t_{1}^{ \pm}$are given by (40).
[1] S. Flügge, Practical Quantum Mechanics (Springer, Berlin, 1999).

[2] A. Mostafazadeh, Generalized unitarity and reciprocity relations for $\mathcal{P} \mathcal{T}$-symmetric scattering potentials, J. Phys. A 47, 505303 (2014).

[3] L. Poladian, Resonance mode expansions and exact solutions for nonuniform gratings, Phys. Rev. E 54, 2963 (1996).

[4] M. Greenberg and M. Orenstein, Irreversible coupling by use of dissipative optics, Opt. Lett. 29, 451 (2004).

[5] M. Kulishov, J. M. Laniel, N. Belanger, J. Azana, and D. V. Plant, Nonreciprocal waveguide Bragg gratings, Opt. Exp. 13, 3068 (2005).

[6] Z. Lin, H. Ramezani, T. Eichelkraut, T. Kottos, H. Cao, and D. N. Christodoulides, Unidirectional Invisibility Induced by PT-Symmetric Periodic Structures, Phys. Rev. Lett. 106, 213901 (2011).

[7] A. Regensburger, C. Bersch, M. A. Miri, G. Onishchukov, D. N. Christodoulides, and U. Peschel, Parity-time synthetic photonic lattices, Nature (London) 488, 167 (2012).

[8] A. Mostafazadeh, Invisibility and PT symmetry, Phys. Rev. A 87, 012103 (2013).

[9] S. A. R. Horsley, M. Artoni, and G. C. La Rocca, Spatial Kramers-Kronig relations and the reflection of waves, Nat. Photonics 9, 436 (2015).

[10] S. Longhi, Wave reflection in dielectric media obeying spatial Kramers-Kronig relations, Europhys. Lett. 112, 64001 (2015).

[11] S. Longhi, Invisibility in $\mathcal{P} \mathcal{T}$-symmetric complex crystals, J. Phys. A 44, 485302 (2011).

[12] R. Uzdin and N. Moiseyev, Scattering from a waveguide by cycling a non-Hermitian degeneracy, Phys. Rev. A 85, 031804 (2012).

[13] H. F. Jones, Analytic results for a PT-symmetric optical structure, J. Phys. A 45, 135306 (2012).

[14] A. Mostafazadeh, Transfer matrices as nonunitary $S$ matrices, multimode unidirectional invisibility, and perturbative inverse scattering, Phys. Rev. A 89, 012709 (2014).
[15] F. Loran and A. Mostafazadeh, Transfer matrix formulation of scattering theory in two and three dimensions, Phys. Rev. A 93, 042707 (2016).

[16] F. Loran and A. Mostafazadeh, Unidirectional invisibility and nonreciprocal transmission in two and three dimensions, Proc. R. Soc. A 472, 20160250 (2016).

[17] A. Mostafazadeh, Spectral Singularities of Complex Scattering Potentials and Infinite Reflection and Transmission Coefficients at Real Energies, Phys. Rev. Lett. 102, 220402 (2009).

[18] L. L. Sánchez-Soto, J. J. Monzóna, A. G. Barriuso, and J. F. Cariñena, The transfer matrix: A geometrical perspective, Phys. Rep. 513, 191 (2012).

[19] M. V. Berry, Lop-sided diffraction by absorbing crystals, J. Phys. A 31, 3493 (1998).

[20] This result generalizes to oblique incident waves with incidence angle $\theta_{0}$ and wave number $\mathbf{k}_{0}=\left(\varpi\left(p_{0}\right), p_{0}\right)$, where $p_{0}:=$ $k \sin \theta_{0}$. A nonzero value of $p_{0}$ has the effect of replacing $n \alpha$ by $n \alpha+p_{0}$ in Eqs. (31) and (34). This in turn implies that the potential $v_{1}(x) e^{i \alpha y} \chi_{a}(x)$ is invisible for the oblique waves provided that $k<\alpha /\left(1-\sin \theta_{0}\right)$.

[21] It is easy to see that $\theta_{n}^{ \pm}$satisfy $\phi_{n}<\theta_{n}^{+} \leqslant \pi / 2 \leqslant \theta_{n}^{-}<\pi-$ $\phi_{n}$, where $\phi_{n}:=\arcsin (n /[N(k)+1])$. Therefore the scattered plane-wave pairs propagate along directions that lie in the wedge-shaped region $\phi_{1}<\theta<\pi-\phi_{1}$.

[22] A. Guo, G. J. Salamo, D. Duchesne, R. Morandotti, M. VolatierRavat, V. Aimez, G. A. Siviloglou, and D. N. Christodoulides, Observation of $\mathcal{P} \mathcal{T}$-Symmetry Breaking in Complex Optical Potentials, Phys. Rev. Lett. 103, 093902 (2009).

[23] A. Mostafazadeh and M. Sarısaman, Lasing-threshold condition for oblique TE and TM modes, spectral singularities, and coherent perfect absorption, Phys. Rev. A 91, 043804 (2015).

[24] L. Ge, H. Cao, and A. D. Stone, Condensation of thresholds in multimode microlasers, Phys. Rev. A 95, 023842 (2017).

[25] F. Loran and A. Mostafazadeh, Perfect broadband invisibility in isotropic media with gain and loss, Opt. Lett. 42, 5250 (2017). 